Check for updates

Cite this: RSC Adv., 2019, 9, 30937

Received 21st August 2019

Accepted 25th September 2019

DOI: $10.1039 / c 9 r a 06583 k$

rsc.li/rsc-advances

\section{Construction of a flexible covalent organic framework based on triazine units with interesting photoluminescent properties for sensitive and selective detection of picric acid $\dagger$}

\begin{abstract}
Yanjie Li, ${ }^{a}$ Yanan Han, ${ }^{a}$ Minghui Chen, ${ }^{a}$ Yaqing Feng ${ }^{\text {ab }}$ and Bao Zhang (DD *a
Highly emissive two-dimensional (2D) covalent organic frameworks (COFs) have rarely been reported due to the challenge of inhibiting the aggregation-caused quenching (ACQ) caused by $\pi-\pi$ stacking between layers. To address this issue, the use of flexible building units is a promising strategy. However, currently reported flexible 2D COFs generally exhibit poor crystallinity, low surface area, etc. and the mechanism of the excellent fluorescence performance for the flexible 2D COFs still needs to be further explored. In this article, a novel flexible 2D COF (DTZ-COF) was synthesized using two $\pi$-electron deficient triazine monomers rather than the commonly used one $\pi$-electron rich fused aromatic ring under solvothermal conditions. Fortunately, DTZ-COF exhibits excellent crystallinity and high surface area $\left(1276.5131 \mathrm{~m}^{2} \mathrm{~g}^{-1}\right.$ and $2087.5502 \mathrm{~m}^{2} \mathrm{~g}^{-1}$ for Brunauer-Emmett-Teller (BET) and Langmuir surface area, respectively), which are rarely observed in previously reported flexible 2D COFs. The increased Lewis basic sites endow DTZ-COF with certain advantages in the separation of $\mathrm{CO}_{2}$ and $\mathrm{N}_{2}$. The comparison between flexible DTZ-COF and a non-flexible 2D COF (TPT-TPT-COF) with a similar framework suggests that the introduction of flexible building units can indeed improve the photoluminescence (PL) efficiency. The $\pi$ electron deficient DTZ-COF has excellent fluorescence performance and exhibits unique solvent responsiveness, such as fluorescence enhancement in aromatic solvents, and fluorescence quenching in alcohol and water. As a chemical sensor for detection of picric acid (2,4,6-trinitrophenol, TNP), the efficient fluorescence quenching involving both static and dynamic behaviors ensures high selectivity and sensitivity (ppb level).
\end{abstract}

\section{Introduction}

Covalent organic frameworks (COFs) are an emerging class of porous crystalline polymers constructed via two or three dimensional (2D or 3D) covalent linkages of organic building blocks. They generally have low densities, high surface areas and thermal stabilities and thus find wide applications in catalysis, ${ }^{\mathbf{1 , 2}}$ sensing, ${ }^{3,4}$ energy storage ${ }^{5,6}$ drug delivery, ${ }^{7,8}$ and gas storage and separation, ${ }^{\mathbf{9}, 10}$ since first reported by O. M. Yaghi and co-workers in $2005 .^{11}$ In particular, the extended $\pi$-conjugated polymer backbones and the formation of one dimensional (1D) columnar $\pi$-arrays via the layer stacking in 2D COFs give rise to their interesting optoelectronic properties for use in photovoltaics, photodetectors, sensors etc., among which

${ }^{a}$ School of Chemical Engineering and Technology, Tianjin University, Tianjin, 300072, P. R. China. E-mail: baozhang@tju.edu.cn

${ }^{b}$ Collaborative Innovation Center of Chemical Science and Engineering, Tianjin, 300072, P. R. China

$\dagger$ Electronic supplementary information (ESI) available. See DOI: $10.1039 / \mathrm{c} 9 \mathrm{ra} 06583 \mathrm{k}$ chemical sensing via 2D COFs has attracted considerable attentions in recent years.

However, due to the aggregation-caused photoluminescence (PL) quenching (ACQ) by the strong $\pi-\pi$ stacking interactions in the highly crystalline bulk 2D COFs, and/or the non-radiative decay resulted from the thermal dissipation via rotationally labile linkages such as imine, highly emissive 2D COFs are limited. ${ }^{12}$ In 2008, D. L. Jiang and co-workers synthesized the first luminescent 2D COF (TP-COF) by the condensation reaction of hydrazine and triphenylene. ${ }^{13}$ Since then, various strategies have been designed to obtain 2D COFs with high fluorescence performance. For example, a strategy involving the aggregation-induced emission (AIE) building units and fluorescent pyrene constructed via the boronate ester linkage and imine linkage has been developed for highly emissive 2D COFs. ${ }^{14}$ Further, non-planar building units were employed to reduce the $\pi-\pi$ interaction during the formation of $2 \mathrm{D}$ COFs. ${ }^{12}$ The bulky organic groups, such as tert-butyl, were attached to the backbone of the frameworks to adjust the $\pi-\pi$ interaction between the layers and increase the PL efficiency. ${ }^{15}$ Apart from the above-mentioned structure tuning of the building blocks for 
highly emissive 2D COFs, physical strategies involving exfoliation of the aggregated $\pi$-stacked COFs layers were also developed resulting in excellent luminescent $2 \mathrm{D}$ covalent organic nanosheets (CONs). ${ }^{\mathbf{1 6}}$

Recently, flexible molecules were attempted as building blocks to construct the luminescent 2D COFs. ${ }^{17}$ However, the flexible 2D COFs that have been reported so far generally exhibit poor crystallinity and low surface areas, since the introduction of flexible building blocks is unfavourable for their orderly extension within the intra- and interlayers of the framework. ${ }^{18-22}$ Thus, the synthesis of the flexible 2D COFs is challenging. Furthermore, the reported flexible 2D COFs are still rare, and so far all of them constructed via the combination of one unit of 2,4,6-tris(4-formylphenoxy)-1,3,5-triazine and one aromatic diamine leading to a donor-acceptor (D-A) type building block involving one $\pi$-electron rich moiety (aromatic rings interconnected with $\mathrm{O}$ and $\mathrm{N}$ donor Lewis basic sites) and one $\pi$ electron deficient core (triazine). The correlation between the photophysical properties and the type of the framework building units still needs to be further explored.

Herein, we designed and synthesized a novel 2D COF (DTZCOF) by the condensation of one flexible 2,4,6-tris(4formylphenoxy)-1,3,5-triazine (TPOT-CHO) unit and one rigid 2,4,6-tris(4-aminophenyl)-1,3,5-triazine (TPT- $\mathrm{NH}_{2}$ ) unit under solvothermal condition, in which two $\pi$-electron deficient triazine cores were introduced leading to an $\mathrm{A}-\pi-\mathrm{A}$ building block. It is expected that with a greater number of electron deficient and basic triazine moieties introduced, the sensitivity of the "guest-host" interaction, in particular for the electron rich/or acidic "guest", can be improved.

Encouragingly, a solid product with good crystallinity and high surface area was obtained. The involvement of the flexible monomer (TPOT-CHO) allowed DTZ-COF to exhibit a good fluorescence performance, even without electron rich aromatic rings. By comparing with a non-flexible $2 \mathrm{D}$ COF with a similar framework, we demonstrated that the increasing of the flexibility of the COFs backbone significantly enhanced the PL properties of the COF materials. Thus, the prepared DTZ-COF with excellent $\mathrm{CO}_{2}$ absorption performance was applied to sense picric acid (2,4,6-trinitrophenol, TNP), which is a highly explosive energy source. ${ }^{23-26}$ A highly selective and sensitive fluorescent detection of TNP in THF was resulted (ppb level).

\section{Experimental}

\subsection{Reagents and chemicals}

Unless otherwise stated, the reagents and solvents used in the synthesis and testing procedures were commercially available and required no further purification. Methanol $(\mathrm{MeOH})$, ethanol (EtOH), chloroform $\left(\mathrm{CHCl}_{3}\right)$, acetonitrile $(\mathrm{MeCN})$, tetrahydrofuran (THF), dioxane, $N, N$-dimethylformamide (DMF), acetone, toluene, $o$-xylene, $m$-xylene, $p$-xylene, mesitylene, chlorobenzene, $o$-dichlorobenzene (o-DCB), benzyl alcohol, $n$-butanol, acetic acid $(\mathrm{AcOH})$, nitrobenzene (NB), 2nitrotoluene (2-NT), 2-nitrophenol (2-NP), 1-methyl-2,6dinitrobenzene (2,6-DNT), 4-cyanoaniline, cyanuric chloride, 4-hydroxybenzaldehyde were purchased from local chemical companies. 2,4,6-Trinitrophenol (TNP) was purchased from J\&K Chemical Ltd. (Beijing, China).

\subsection{Instruments}

Powder X-ray diffraction (PXRD) patterns were collected using a D/Max-2500 X-ray diffractometer with $\mathrm{Cu}-\mathrm{K} \alpha$ radiation. Fourier-transform infrared (FT-IR) spectra was recorded on a Nicolet is 50 ATR-FTIR instrument. Thermogravimetric analysis (TGA) was carried out on a Delta Series TA-SDTQ600 analyzer in nitrogen atmosphere using aluminum crucibles. The solid phase ${ }^{13} \mathrm{C}-\mathrm{NMR}$ spectra were obtained on a Varian 300 $\mathrm{MHz}$ solid state Infinityplus $300 \mathrm{NMR}$ spectrometer. The Brunauer-Emmett-Teller (BET) surface areas were screened by nitrogen adsorption and desorption at $77 \mathrm{~K}$ using ASAP-2020. Pore size distributions and pore volumes were derived from the adsorption branches of the isotherms using the density functional theory (DFT) pore model. Scanning electron microscopies (SEM) were carried out using a Hitachi SU3500 SEM instrument. The fluorescence excitation and emission spectra were performed on an G9800A fluorescence spectrophotometer (Agilent Technologies, USA) with the slit widths both set at $5 \mathrm{~nm}$. The fluorescence lifetime was carried out with an Fluorolog-3 fluorescence spectrophotometer (HORIBA Jobin Yvon, USA). Atomic Force Microscope (AFM) was conducted on Dimension icon of Bruker. The geometry optimization of lattice structure and refinement of PXRD pattern were performed at forcite module and reflex module in Material Studio, respectively.

\subsection{Synthesis of DTZ-COF}

The monomers (TPT- $\mathrm{NH}_{2}$ and TPOT-CHO) were synthesized using 4-aminobenzonitrile and 2,4,6-trichloro-1,3,5-triazine as starting materials (Fig. 1), respectively, according to the methods reported in the literatures. ${ }^{17,27}$

Then DTZ-COF was prepared by the condensation of TPOTCHO and TPT- $\mathrm{NH}_{2}$. A $10 \mathrm{~mL}$ Pyrex tube was charged with TPT-NH ${ }_{2}$ (10.6 mg, $0.03 \mathrm{mmol}$ ), TPOT-CHO (13.2 mg, 0.03 $\mathrm{mmol}), o$-DCB (0.5 mL), $n$-butanol $(0.5 \mathrm{~mL})$ and AcOH $(0.1 \mathrm{~mL}, 6$ $\mathrm{M})$. The mixture was sonicated for 15 minutes, degassed through three freeze-pump-thaw cycles and sealed under vacuum, successively. The reaction was heated at $120^{\circ} \mathrm{C}$ for $72 \mathrm{~h}$

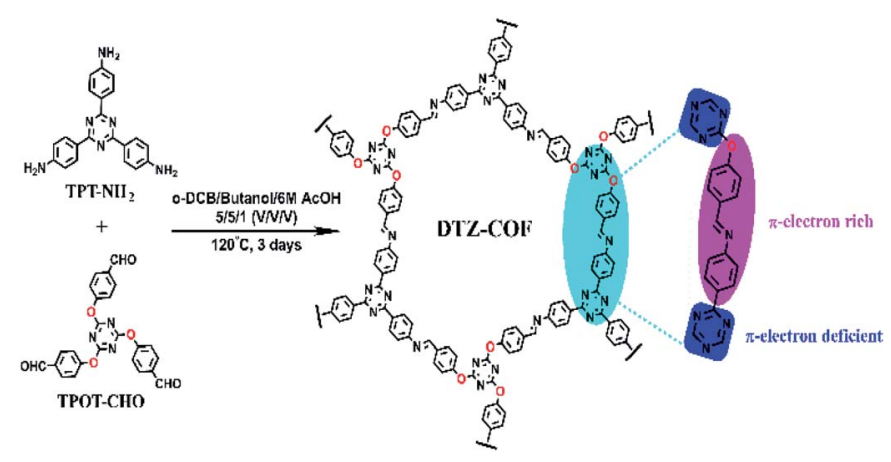

Fig. 1 Schematic diagram of solvothermal synthesis of DTZ-COF. 
yielding a yellow solid at the bottom of the tube which was isolated by centrifugation, washed with THF using Soxhlet extraction for $24 \mathrm{~h}$, and dried under vacuum at $75^{\circ} \mathrm{C}$ for $10 \mathrm{~h}$ to afford yellow powder for various tests.

\subsection{Solvent responsiveness test}

$5 \mathrm{mg}$ of DTZ-COF was added to $50 \mathrm{~mL}$ of the solvent to be examined and sonicated for $15 \mathrm{~min}$ to obtain a uniform dispersion. Then $2.5 \mathrm{~mL}$ of the suspension was added to the cuvette $(1 \mathrm{~cm} \times 1 \mathrm{~cm})$ and excited upon $350 \mathrm{~nm}$.

\subsection{Fluorescence detection of TNP}

The detection of TNP by DTZ-COF was carried out in THF dispersion $\left(0.1 \mathrm{mg} \mathrm{mL} \mathrm{mL}^{-1}\right)$. The effect of different concentration of TNP on the PL of DTZ-COF was investigated by measuring the change in fluorescence intensity $(485 \mathrm{~nm})$. The concentration of TNP was increased gradually from 0 to $10.7 \mathrm{ppm}$.

\section{Results and discussion}

\subsection{Characterization of DTZ-COF}

DTZ-COF was prepared by the condensation reaction of TPT$\mathrm{NH}_{2}$ and TPOT-CHO under the catalysis of acetic acid. The FTIR spectra shows that the vibration peaks of $\mathrm{C}=\mathrm{O}$ and $\mathrm{N}-\mathrm{H}$ tend to disappear, and a new stretching vibration peak belonging to the imine bond $(\mathrm{C}=\mathrm{N})$ appear at $1621 \mathrm{~cm}^{-1}$, which proves the occurrence of the condensation reaction (Fig. 2a). The TG curve indicates that DTZ-COF can maintain structural stability at a high temperature of $350{ }^{\circ} \mathrm{C}$ (Fig. 2b). The solid phase ${ }^{13} \mathrm{C}-\mathrm{NMR}$ spectrum of DTZ-COF exhibits signals at $158.7 \mathrm{ppm}$ and 174.1 ppm which indicate the presence of imine and triazene functionalities, respectively (Fig. 2c). The SEM image shows that DTZ-COF prepared by the solvothermal method exhibits a belt appearance (Fig. S4†).
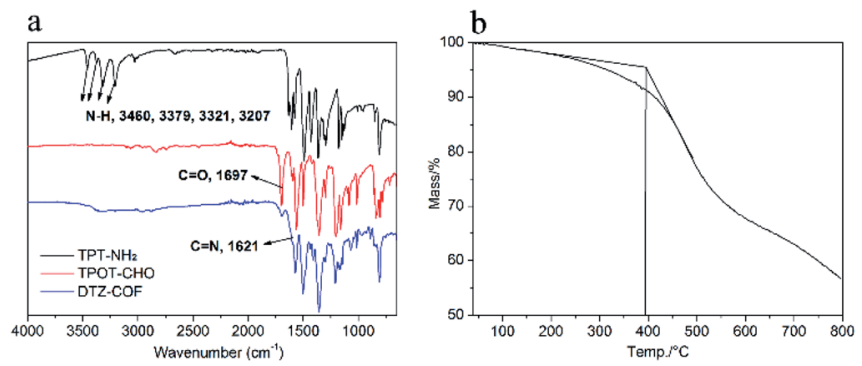

c
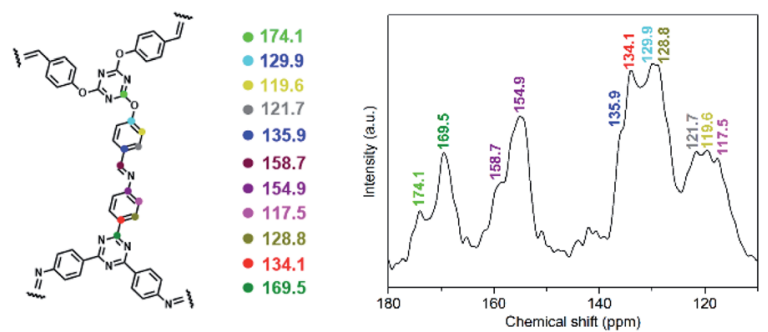

Fig. 2 The FT-IR (a), TGA (b) and solid phase ${ }^{13} \mathrm{C}-\mathrm{NMR}$ (c) spectra of DTZ-COF.
PXRD analysis was performed to determine the crystalline structure of DTZ-COF (Fig. 3a). The PXRD pattern displays five prominent diffraction peaks at $2 \theta=4.02^{\circ}, 6.92^{\circ}, 8.02^{\circ}, 10.62^{\circ}$, and $25.19^{\circ}$, which can be assigned to the (100), (110), (200), (210), and (001) facets, respectively. The Pawley-refined PXRD profile of AA stacking matched with the experimental pattern very well with $R_{\mathrm{wp}}$ $=5.68 \%$ and $R_{\mathrm{p}}=4.51 \%$. DTZ-COF adopts the AA stacking mode of a space group of $P 3$ with the optimized parameters of $a=b=$ $26.8882 \AA$ and $c=3.6611 \AA$ A $\alpha=\beta=90^{\circ}$ and $\gamma=120^{\circ}$ (Fig. 3b). Nitrogen sorption isotherms were performed at $77 \mathrm{~K}$ to investigate the porosity of DTZ-COF and the adsorption profiles are characteristic type I isotherm indicating a microporous structure (Fig. 3c). The BET surface area was calculated to be $1276.5131 \mathrm{~m}^{2} \mathrm{~g}^{-1}$, while the Langmuir model gave a surface area of $2087.5502 \mathrm{~m}^{2} \mathrm{~g}^{-1}$, and the pore volume was determined to be $0.82 \mathrm{~cm}^{3} \mathrm{~g}^{-1}$ at $P / P^{0}=0.95$. To the best of our knowledge, the surface area of DTZ-COF is much greater than those flexible 2D COFs reported so far. DFT pore model shows that the pore size distribution is mainly concentrated at $2.1 \mathrm{~nm}$, which is consistent with the value of $d$-spacing obtained from PXRD profiles at $2 \theta=4.02^{\circ}(2.2 \mathrm{~nm}$ for DTZ-COF). Considering the more Lewis basic sites introduced in the frameworks, the as-synthesized DTZ-COF was further employed as the adsorbent for $\mathrm{CO}_{2}$ capture, the $\mathrm{CO}_{2}$ and $\mathrm{N}_{2}$ adsorption isotherms were collected at $273 \mathrm{~K}$ and $298 \mathrm{~K}$ under 1.0 bar pressure (Fig. 3d). The amounts of $\mathrm{CO}_{2}$ uptake steadily increased with the increasing pressure and reached the maximum values of 31.1 and $21.2 \mathrm{~cm}^{3} \mathrm{~g}^{-1}$ at 273 and $298 \mathrm{~K}$, respectively. The low amounts of $\mathrm{N}_{2}$ uptake under the same conditions indicate that DTZ-COF has a good selective uptake of $\mathrm{CO}_{2}$ and could be applied to the separation of $\mathrm{CO}_{2}$ and $\mathrm{N}_{2}$.

\subsection{Photophysical properties}

To study how the flexible building units influence the PL of $2 \mathrm{D}$ COFs, the non-flexible 2D COF involving two rigid building units TPT- $\mathrm{NH}_{2}$ and 2,4,6-tris(4-formylphenyl)-1,3,5-triazine

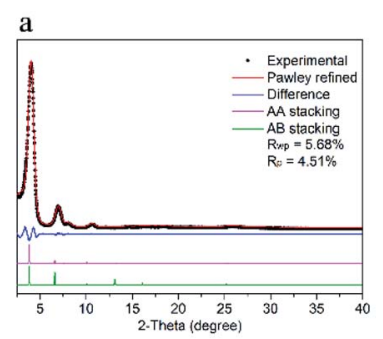

b
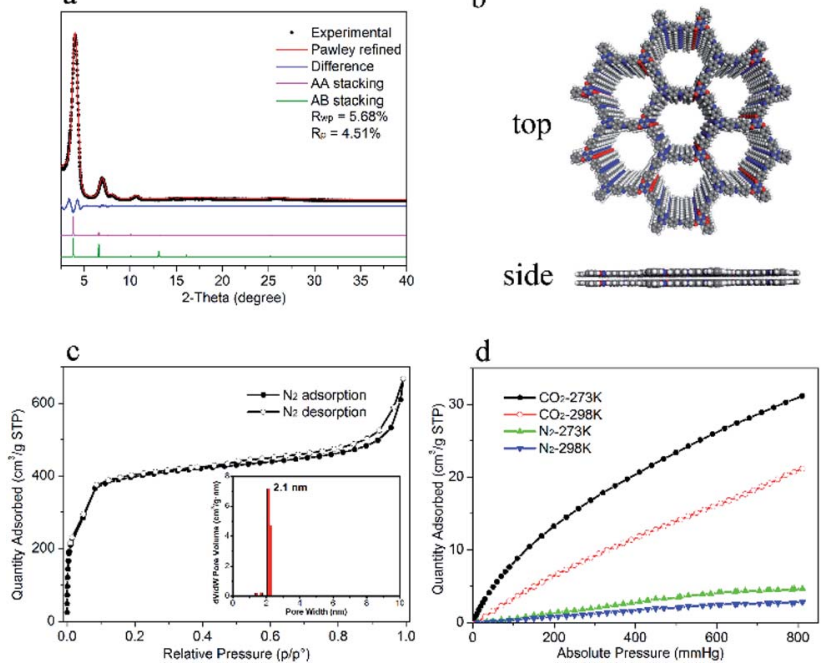

Fig. 3 (a) PXRD patterns of DTZ-COF. (b) The top view and side view of AA stacking mode of DTZ-COF. (c) Nitrogen sorption isotherms of DTZ-COF. Inset: pore size distribution of DTZ-COF. (d) $\mathrm{CO}_{2}$ and $\mathrm{N}_{2}$ adsorption isotherms of DTZ-COF at $273 \mathrm{~K}$ and $298 \mathrm{~K}$. 
(TPT-CHO) with a similar framework structure reported by S. W. Kuo and co-workers was synthesized leading to TPT-TPT-COF (Fig. S7 $\dagger$ ). ${ }^{27}$ The fluorescence excitation and emission spectra of DTZ-COF and TPT-TPT-COF in THF dispersions were examined under the same conditions (Fig. S9 and S10 $\dagger$ ). DTZ-COF showed an emission band at $485 \mathrm{~nm}$ upon excited at $350 \mathrm{~nm}$ and the PL intensity reached about four times that of TPT-TPTCOF, of which the maximum emission band appeared at $468 \mathrm{~nm}$ upon excited at same wavelength. And the THF dispersion of DTZ-COF exhibited a strong yellow luminescence under UV-light irradiation of $365 \mathrm{~nm}$, while TPT-TPT-COF showed almost no visible fluorescence emission (inset of Fig. 4a). These results indicate that DTZ-COF bearing flexible building units exhibits stronger fluorescence emission, compared with the rigid TPT-TPT-COF, which suggested the flexible TPOT-CHO building unit introduced into DTZ-COF can partially release the ACQ effect leading to the strong fluorescence emission of DTZ-COF. It was also found that the absorption band of DTZ-COF (393 nm) powder is significantly blue-shifted compared to that of solid TPT-TPT-COF (423 nm) (Fig. 4b), indicative of the stronger $\pi-\pi$ interaction present in TPT-TPT-COF.

The PXRD diffraction peak of (001) reflection for DTZ-COF and TPT-TPT-COF appears at $2 \theta=25.19^{\circ}$ and $25.56^{\circ}$, respectively, the calculated layer-spacing of DTZ-COF $(3.55 \AA)$ is wider compared with TPT-TPT-COF (3.48 $\AA$ ) referencing to Bragg's law. Furthermore, according to the theoretically simulated crystal structure of DTZ-COF and TPT-TPT-COF, the cell-lengthc value of DTZ-COF (3.66 $\AA$ ) is increased by $0.15 \AA$ compared to TPT-TPT-COF (3.51 $\AA$ ), indicating the weakened $\pi-\pi$ stacking in
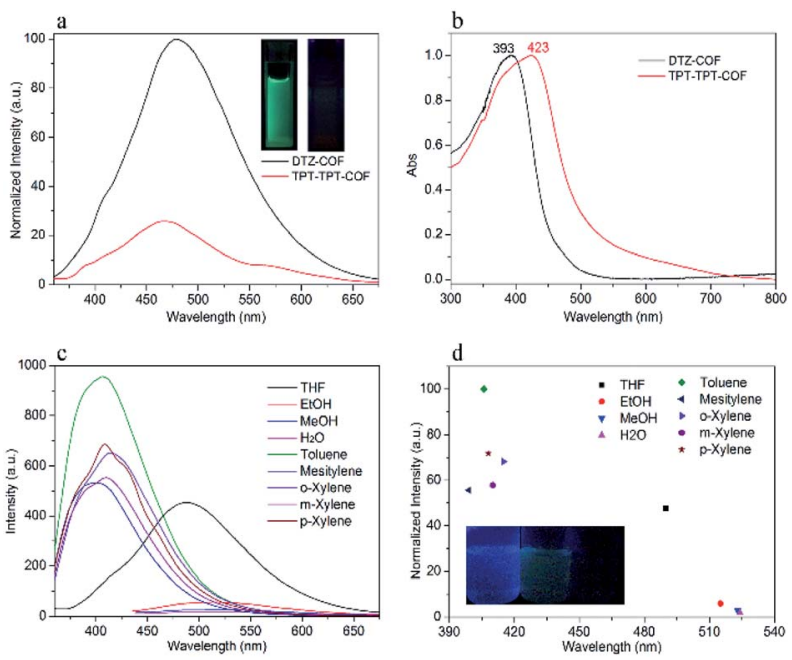

Fig. 4 (a) Normalized fluorescence emission spectra of DTZ-COF and TPT-TPT-COF in THF dispersions $\left(\lambda_{\mathrm{ex}}=350 \mathrm{~nm}\right)$. Inset: images of DTZ-COF (left) and TPT-TPT-COF (right) in THF dispersions under UVlight irradiation of $365 \mathrm{~nm}$. (b) Solid-state UV-vis spectra for DTZ-COF and TPT-TPT-COF. (c) The fluorescence emission spectra of DTZCOF dispersed in different solvents $\left(\lambda_{\mathrm{ex}}=350 \mathrm{~nm}\right)$. (d) The wavelength and intensity of the fluorescence emission of DTZ-COF in different solvents ( $\lambda_{\mathrm{ex}}=350 \mathrm{~nm}$ ). Inset: images of DTZ-COF in toluene, THF and $\mathrm{H}_{2} \mathrm{O}$ (from left to right) dispersions under UV-light irradiation of $365 \mathrm{~nm}$.
DTZ-COF due to the presence of the flexible building unit. The overall results above suggested that the increment of framework flexibility inhibited the $\pi-\pi$ stacking between layers and overcame the ACQ effect, thereby increasing the PL efficiency of the resultant COFs.

Interestingly, it was found that the PL of DTZ-COF showed solvent responsiveness exhibiting varying maximum emission wavelengths and intensities in different solvents when excited at the same wavelength (Fig. 4c and Fig. S11 $\dagger$ ). The dispersions of DTZ-COF in aromatic solvents showed varying degrees of blue shift and the maximum blue shift of $90 \mathrm{~nm}$ occurred in mesitylene compare to THF. Furthermore, the fluorescence intensity enhancement also occurred in the dispersions of aromatic solvents which is believed to be attributed to the charge transfer between the electron-rich aromatic ring of the solvent and the electron-deficient triazine cores of DTZ-COF. Thus, it can be observed that the fluorescence intensity of the toluene dispersion is about twice that of the THF dispersion. Interestingly, unlike the results reported by S. K. Mandal and coworkers, ${ }^{17}$ we observed that alcohol and water almost completely quenched the fluorescence emission, which probably can be ascribed to a ground state complex formed between DTZ-COF and these solvent molecules via hydrogen bonding (Fig. 4d).

\subsection{Fluorescence detection of TNP}

Due to the high surface area and porosity, as well as the strong fluorescent properties of DTZ-COF, it can thus be exploited as fluorescence sensors for the detection of explosives. We then examined the effect of TNP on the fluorescence emission of DTZ-COF in THF dispersion and it was shown that TNP can quench over $90 \%$ of the fluorescence intensity at a concentration of 10.7 ppm. The TNP embedded in DTZ-COF was removed by centrifugation, and the obtained COF material recycled for the detection of TNP which showed that DTZ-COF could still maintain a high sensitivity to TNP after four cycles (Fig. S13 $\dagger$ ). To determine the sensing selectivity of DTZ-COF for TNP, the fluorescence quenching experiments were also performed on several non-explosive materials with similar structures, including 2-NP, 2,6-DNT, NB and 2-NT (Fig. 5a). The slight attenuation of fluorescence intensity upon addition of the contrast $(18.6 \%$ for $2-\mathrm{NP}, 11.5 \%$ for $2,6-\mathrm{DNT}, 6.7 \%$ for NB and $1.8 \%$ for $2-\mathrm{NT}$ ) suggested that DTZ-COF has excellent target selectivity for TNP. The fluorescence emission spectra of DTZCOF in THF dispersion was examined upon varying the concentration of TNP (Fig. 5b). As observed, when the concentration of TNP gradually increases, the fluorescence intensity of the dispersion exhibits a gradient weakening. The results have shown that trace amounts of TNP could dramatically reduce the fluorescence intensity of DTZ-COF, and DTZ-COF could be applied to the visible fluorescence detection of TNP.

Next, the quenching mechanism was evaluated from the Stern-Volmer constant,

$$
\begin{gathered}
I_{0} / I=1+K_{\mathrm{SV}}[\mathrm{Q}] \\
K_{\mathrm{SV}}=K_{\mathrm{Q}} \tau
\end{gathered}
$$





Fig. 5 (a) Fluorescence quenching efficiency of different nitroaromatic compounds towards DTZ-COF at a concentration of $10.71 \mathrm{ppm}$. (b) The fluorescence emission spectra of DTZ-COF in THF dispersion upon increasing the concentration of TNP (0 to $10.7 \mathrm{ppm}$ ). (c) Stern-Volmer plot for fluorescence quenching of DTZ-COF by TNP at low concentrations. (d) Fluorescence lifetime decay profiles of the THF dispersions of DTZ-COF in presence of TNP $\left(\lambda_{\mathrm{ex}}=350 \mathrm{~nm}\right.$ and $\lambda_{\mathrm{em}}=485 \mathrm{~nm}$ ). (e) Schematic diagram of fluorescent quenching process caused by TNP.

where $I_{0}$ and $I$ represent the fluorescence intensities of the blank sample and the quencher-added sample, respectively. $K_{\mathrm{SV}}$ is the quencher coefficient, and $[\mathrm{Q}]$ is the molar concentration of quencher. $K_{\mathrm{Q}}$ is the quenching rate constant, and $\tau$ is the fluorescence lifetime of the blank sample. ${ }^{28}$ It was found that at low concentrations of TNP, a near linear Stern-Volmer plot was obtained with a $K_{\mathrm{SV}}$ value of $0.38 \mathrm{ppm}^{-1}\left(8.71 \times 10^{4} \mathrm{M}^{-1}\right)$ and $K_{\mathrm{Q}}$ calculated to be $1.15 \times 10^{8} \mathrm{ppm}^{-1} \mathrm{~s}^{-1}\left(2.62 \times 10^{13} \mathrm{M}^{-1} \mathrm{~s}^{-1}\right)$ (Fig. 5c). The $K_{\mathrm{SV}}$ and $K_{\mathrm{Q}}$ values are much greater than those reported for MOF based sensors, ${ }^{29}$ and comparable to the stateof-the-art COF based ones reported, ${ }^{17}$ indicating the strong interaction between DTZ-COF and TNP. The detection limit of DTZ-COF for TNP was calculated to be as low as $81.8 \mathrm{ppb}$ (3.57 $\times 10^{-7} \mathrm{M}$ ). The Stern-Volmer plot of TNP showed linearity at lower concentrations and bent upwards at higher concentrations (Fig. S15†), which suggested that the quenching process was caused by both static and dynamic behaviour involving TNP. ${ }^{30}$

To further investigate the quenching process of DTZ-COF in the presence of TNP, we examined the fluorescence lifetime of the THF dispersion of DTZ-COF (Fig. 5d). DTZ-COF exhibited a biexponential decay profile with an average lifetime value of
$3.32 \mathrm{~ns}$. And the average lifetime exhibited a trivial attenuation (3.00 ns) after addition of TNP (10.7 ppm), indicative of a combination of static and dynamic quenching. Thus, it was proposed that the TNP molecule as a hydrogen bond donor can form a ground state complex via hydrogen bonding with the exposed $\mathrm{N}$ and $\mathrm{O}$ atoms on the skeleton resulting in the quenching of the fluorescence, as shown in Fig. $5 \mathrm{e} \cdot{ }^{31}$ Moreover, as reported in previous literatures, ${ }^{32}$ the conduction band (CB) of COFs is usually higher in energy than the LUMO of TNP, thus the excited electron of DTZ-COF is transferred to TNP, which could lead to fluorescence quenching.

\section{Conclusions}

In summary, a novel triazine-based 2D COF was designed and synthesized by the condensation reaction of $\pi$-electrondeficient triazine monomers TPT- $\mathrm{NH}_{2}$ and TPOT-CHO under solvothermal condition. Interestingly, DTZ-COF possesses good crystallinity and high surface area that have rarely been observed in previously reported flexible $2 \mathrm{D} \mathrm{COFs}$. The excellent fluorescence performance of DTZ-COF suggested that the introduction of flexible building units could enhance the PL efficiency and provided a new strategy for the construction of highly emissive 2D COFs. DTZ-COF exhibits unique solvent responsiveness with varying wavelength and intensity of the fluorescence emission in different solvents. The efficient fluorescence quenching involving both static and dynamic behaviour allows DTZ-COF to detect TNP with high sensitivity and selectivity (ppb level). Due to the encouraging properties of the newly developed flexible 2D DTZ-COF involving two $\pi$-electron deficient triazine cores, such as unprecedented high surface area of this kind and excellent emissive performance, further utility exploration is still in progress.

\section{Conflicts of interest}

There are no conflicts to declare.

\section{Acknowledgements}

This work was supported by the National Natural Science Foundation of China (No. 21761132007).

\section{Notes and references}

1 S. Y. Ding, J. Gao, Q. Wang, Y. Zhang, W. G. Song, C. Y. Su and W. Wang, J. Am. Chem. Soc., 2011, 133, 19816-19822.

2 H. Vardhan, L. X. Hou, E. Yee, A. Nafady, M. A. AlAbdrabalnabi, A. M. Al-Enizi, Y. X. Pan, Z. Y. Yang and S. Q. Ma, ACS Sustainable Chem. Eng., 2019, 7, 4878-4888.

3 G. Q. Lin, H. M. Ding, D. Q. Yuan, B. S. Wang and C. Wang, J. Am. Chem. Soc., 2016, 138, 3302-3305.

4 S. Y. Ding, M. Dong, Y. W. Wang, Y. T. Chen, H. Z. Wang, C. Y. Su and W. Wang, J. Am. Chem. Soc., 2016, 138, 30313037. 
5 S. Wang, Q. Y. Wang, P. P. Shao, Z. Y. Han, X. Gao, L. Ma, S. Yuan, X. J. Ma, J. W. Zhou, X. Feng and B. Wang, J. Am. Chem. Soc., 2017, 139, 4258-4261.

6 C. R. Mulzer, L. X. Shen, R. P. Bisbey, J. R. McKone, N. Zhang, H. D. Abruna and W. R. Dichtel, ACS Cent. Sci., 2016, 2, 667673.

7 L. Y. Bai, S. Z. F. Phua, W. Q. Lim, A. Jana, Z. Luo, H. P. Tham, L. Z. Zhao, Q. Gao and Y. L. Zhao, Chem. Commun., 2016, 52, 4128-4131.

8 V. S. Vyas, M. Vishwakarma, I. Moudrakovski, F. Haase, G. Savasci, C. Ochsenfeld, J. P. Spatz and B. V. Lotsch, Adv. Mater., 2016, 28, 8749-8754.

9 S. B. Kalidindi and R. A. Fischer, Phys. Status Solidi B, 2013, 250, 1119-1127.

10 Y. F. Zeng, R. Q. Zou and Y. L. Zhao, Adv. Mater., 2016, 28, 2855-2873.

11 A. P. Cote, A. I. Benin, N. W. Ockwig, M. O'Keeffe, A. J. Matzger and O. M. Yaghi, Science, 2005, 310, 1166-1170.

12 Q. Gao, X. Li, G. H. Ning, K. Leng, B. B. Tian, C. B. Liu, W. Tang, H. S. Xu and K. P. Loh, Chem. Commun., 2018, 54, 2349-2352.

13 S. Wan, J. Guo, J. Kim, H. Ihee and D. L. Jiang, Angew. Chem., Int. Ed., 2008, 47, 8826-8830.

14 S. Dalapati, E. Jin, M. Addicoat, T. Heine and D. L. Jiang, J. Am. Chem. Soc., 2016, 138, 5797-5800.

15 Z. P. Li, Y. W. Zhang, H. Xia, Y. Mu and X. M. Liu, Chem. Commun., 2016, 52, 6613-6616.

16 G. Das, B. P. Biswal, S. Kandambeth, V. Venkatesh, G. Kaur, M. Addicoat, T. Heine, S. Verma and R. Banerjee, Chem. Sci., 2015, 6, 3931-3939.

17 P. Das and S. K. Mandal, J. Mater. Chem. A, 2018, 6, 1624616256.
18 S. K. Konavarapu and K. Biradha, Cryst. Growth Des., 2019, 19, 362-368.

19 D. Mullangi, V. Dhavale, S. Shalini, S. Nandi, S. Collins, T. Woo, S. Kurungot and R. Vaidhyanathan, Adv. Energy Mater., 2016, 6, 1600110-1600117.

20 Y. Li, C. Wang, S. J. Ma, H. Y. Zhang, J. J. Ou, Y. M. Wei and M. L. Ye, ACS Appl. Mater. Interfaces, 2019, 11, 11706-11714.

21 C. F. Zhao, C. S. Diercks, C. H. Zhu, N. Hanikel, X. K. Pei and O. M. Yaghi, J. Am. Chem. Soc., 2018, 140, 16438-16441.

22 X. H. Guo, Y. Tian, M. C. Zhang, Y. Li, R. Wen, X. Li, X. F. Li, Y. Xue, L. J. Ma, C. Q. Xia and S. J. Li, Chem. Mater., 2018, 30, 2299-2308.

23 X. Z. Song, S. Y. Song, S. N. Zhao, Z. M. Hao, M. Zhu, X. Meng, L. L. Wu and H. J. Zhang, Adv. Funct. Mater., 2014, 24, 4034-4041.

24 Y. X. Ma, H. Li, S. Peng and L. Y. Wang, Anal. Chem., 2012, 84, 8415-8421.

25 Y. Peng, A. J. Zhang, M. Dong and Y. W. Wang, Chem. Commun., 2011, 47, 4505-4507.

26 Y. Wang and Y. N. Ni, Anal. Chem., 2014, 86, 7463-7470.

27 A. F. M. El-Mahdy, C. H. Kuo, A. Alshehri, C. Young, Y. Yamauchi, J. Kim and S. W. Kuo, J. Mater. Chem. A, 2018, 6, 19532-19541.

28 Y. Zhou, J. Y. Yan, N. N. Zhang, D. J. Li, S. Z. Xiao and K. B. Zheng, Sens. Actuators, B, 2018, 258, 156-162.

29 J. D. Xiao, L. G. Qiu, F. Ke, Y. P. Yuan, G. S. Xu, Y. M. Wang and X. Jiang, J. Mater. Chem. A, 2013, 1, 8745-8752.

30 S. S. Nagarkar, B. Joarder, A. K. Chaudhari, S. Mukherjee and S. K. Ghosh, Angew. Chem., Int. Ed., 2013, 52, 2881-2885.

31 R. Gomes and A. Bhaumik, RSC Adv., 2016, 6, $28047-28054$. 32 M. E. Germain and M. J. Knapp, Chem. Soc. Rev., 2009, 38, 2543-2555. 